\title{
CALCULATION METHOD OF HERMETIC SEAL ASSEMBLY PARAMETERS OF THE PACKER USED DURING REPAIRING WELLS
}

\section{МЕТОДИКА РАСЧЕТА ПАРАМЕТРОВ ГЕРМЕТИЗАЦИИ УПЛОТНИТЕЛЬНОГО УЗЛА ПАКЕРА}

\section{Aslanov Jamaladdin Nuraddin ${ }^{1}$ \\ Huseynli Zenfira Seyidi}

DOI: https://doi.org/10.30525/978-9934-588-15-0-49

\begin{abstract}
Increasing the service life of exploitational packers used in oil and gas production by increasing the wear resistance and stability on deformation of its sealing collar is one of the most urgent tasks. From this point of view, the monograph examines the stress-strain state of packer seals. In the research work, different directions of the stress-strain of packer seals were studied. The main of these directions is the research of the deformation state of packer seals based on potential energy functionalities. Besides, the research of the initial deformation on the sealing effect at the time of its landing is one of the problems raised in the research. In the article question the factor determining the sealing ability of the seal downhole packers has been studied. It has been established that $30-40 \%$ of failed jobs associated with ventilated packer. It is also shown that the sealing ability packer seals the equipment determined by a complex mechanical and technological factors. These factors caused the value of internal and contact stresses encountered when using the seal, the size and speed of movement, purity compactibility surface. It was found that the seal provided by the compression deformation of rubber packer elements. The degree of deformation of the sealing member should be sufficient to keep the sealing elements of the installation site of the hood, landing under the action of pressure medium compacted. To determine the initial contact stress created during the defor-
\end{abstract}

\footnotetext{
${ }^{1} \mathrm{PhD}$, Associate Professor, Department «Oil-gas equipment», Azerbaijan State Oil and Industry University, Azerbaijan

${ }^{2} \mathrm{PhD}$, Associate, Department «Oil-gas equipment»,

Azerbaijan State Oil and Industry University, Azerbaijan
} 
mation of the sealing element found the form factor of the seal. Also take into account the effective area of the seal. The analytical expressions for the friction force on the surface contact sealing element and the pressure causes a complete displacement of the seal where the seal is withdrawn from the installation site. Defined the expression of the pressure conditions of stability and seal contact pressure with the long-term operation of the packers (seal assembly) in a given temperature range. The article considers method of calculating the parameters of sealing of the seal assembly of the packer used during the repair of wells. For this purpose using direct variational Ritz methods determined the total potential energy of the packer seal, minimizing the potential energy of the sealing element under the terms of the Euler. Defined variational constant and the parameters of the seal packer.

\section{1. Вступление}

Увеличение срока работоспособности применяемых при добыче нефти и газа эксплуатационных пакеров, за счет повышения износостойкости и устойчивости на деформацию его уплотнительных манжет является одной из самых актуальных задач. С этой точки зрения в монографии исследуется напряженно-деформированное состояние уплотнителей пакера.

В исследовательской работе изучены разные направления напряженной деформации уплотнителей пакера. Основным из этих направлений является исследования деформационной состоянии уплотнителей пакера на основе потенциальных энергетических функционалов.

Кроме того, изучение влияния начальной деформации на уплотнительный эффект в момент его посадки является одной из задач, поднятых в исследовании.

В исследовательской работе рассмотрена методика расчета параметров герметизации уплотнительного узла пакеров. С этой целью используя прямые вариационные методы Ритца изыскано полная потенциальная энергия уплотнителя пакера, минимизирую потенциальной энергии уплотнительного элемента по условиям Эйлера. Определены вариационный постоянный и параметры уплотнителя пакера.

Установлено, что герметизация обеспечивается за счет деформации сжатия резиновых элементов пакера. Степень деформации уплотнительного элемента должна быть достаточной, чтобы удержать уплотнитель- 
ные элементы от вытяжки из места установки-посадки при действии на него давления герметизируемой среды. Для определения начальное контактное напряжение, созданное при деформации уплотнительного элемента найдено коэффициент формы уплотнителя. Также учтена эффективная площадь уплотнения. Получена аналитическая выражения, сила трения на поверхности контакта уплотнительного элемента и значение давления вызывающего полное перемещение уплотнения при котором уплотнитель не вытягивается из места установки.

\section{2. На основе потенциальной энергии изучения расчетной деформации}

Пакерующий узел один из наименее надежных в работе узлов пакерного оборудования. Решающее значение для обеспечения герметичности пакеровки имеет правильный выбор параметров скважины: точного определения фактического диаметра скважины, место установки пакера и оптимального перепада давления на пакер, оптимальные формы и размеры (также осевые деформации) уплотнителя пакера [8].

Анализ промысловых данных работ по пакеровке скважин показывает, что примерно 30-40\% неудачных работ связано с негерметичностью пакеровки [5].

Теоретическими и многочисленными стендовыми исследованиями установлено, что герметизирующая способность уплотнений пакерного оборудования определяется комплексом физико-механических и технологических факторов [9].

К физико-механическим факторам принято относить физико-механические характеристики материала уплотнений. Технологически-напряженное состояние уплотнения характеризуется величиной внутренних и контактных напряжений, возникающих при работе уплотнения, величины и скорости перемещения, чистоты уплотняемой поверхности. В этой связи необходимо отметить, что герметизирующие элементы работают в осложненных условиях, обусловленных высокими гидравлическими давлениями - до $100 \mathrm{MПа,} \mathrm{агрессивностью} \mathrm{рабочей}$ среды и высокими температурами (порядка $200^{\circ}$ и выше). В большинстве случаев герметизирующие элементы подвержены одновременному воздействию перечисленных факторов, что отрицательно сказывается на их работоспособности (рис. 3). Нарушение герметичности 
сопровождается значительными размывами уплотняемых поверхностей, что в ряде случаев приводит к полному их разрушению и невозможности их ремонта и дальнейшего использования [5]. Все это свидетельствует о том, что обеспечение герметичности является одним из основных факторов нормальной работы оборудования в целом. Герметизирующим элементом пакера являются его уплотнительные резиновые элементы. Герметизация обеспечивается за счет деформации сжатия резиновых элементов пакера в осевом направлении, при установке посадки его во внутренней поверхности эксплуатационной колонны.

При расчете уплотнений из несжимаемого резинового материала широко используют прямые методы. Очень плодотворным оказывается применение метода Ритца:

$$
\int_{\Omega} u_{i} p_{i} d \Omega=\Delta \cdot \int_{\Omega} P_{i} d \Omega=-\Delta P=-\Delta Q
$$

где $P=Q$ - осевые усилия приложенное к уплотнителю; $\Delta-$ осевое перемещение деформации уплотнителя пакера; $U_{i}$ - радиальное перемещение; $P_{i}=P=Q-$ осевое усилия; $d \Omega-$ элементарный площадь поверхности уплотнителя.

Функционал полный потенциальной энергии уплотнителя определяется по следующим выражением [1]:

$$
\Pi=G \int_{V}\left(\varepsilon_{r}^{2}+\varepsilon_{\theta}^{2}+\varepsilon_{z}^{2}+\frac{1}{2} \gamma_{r z}^{2}\right) d V-\Delta Q
$$

Выразив $\Delta$ из условия $W=-\Delta \mathrm{W}=-\Delta$ при $z=h+\left(r-R_{2}\right) \operatorname{tg} \beta$ и исключив при помощи условия несжимаемости деформации $\varepsilon_{r}$, подсчитаем полную энергию $П$ :

$$
\Pi=2 \Pi G \int_{R_{\theta H}}^{R_{H}} \int_{\left(r-R_{1}\right) \operatorname{tg} \beta}^{h+\left(r-R_{1}\right) \operatorname{tg} \beta}\left(\varepsilon_{Z}^{2}+\varepsilon_{\theta}^{2}+\varepsilon_{Z} \varepsilon_{\theta}+\frac{1}{2} \gamma_{z r}^{2}\right) \cdot r \cdot d r d z-\Delta Q
$$

где $\varepsilon_{r}=-\left(\varepsilon_{\theta}+\varepsilon_{Z}\right) \varepsilon_{r}, \varepsilon_{\theta}$ и $\varepsilon_{z}$ соответственно относительно радиальная, потенциальная и осевая деформация уплотнителя; $\gamma_{r, z}-$ углевые деформации уплотнителя; G-модуль сдвига; $\Delta$-осевая деформация уплотнителя в точке приложения; $d V$ - элементарный объем уплотнителя; $R_{6}, R_{H}$ - соответственно внутренние и наружные радиусы уплотнителя; $W, z$ - соответственные перемещение в направлении оси $z$.

Для деформации уплотнителя пакера в осевом направлении примем 


$$
W=a_{k}\left(1-\frac{Z^{2}}{h^{2}}\right)
$$

где $a_{\kappa}$ - постоянная, подлежащая определению.

Напишем условие несжимаемости

Из (4) с учетом (5) имеем:

$$
\varepsilon_{r}=\varepsilon_{\theta}+\varepsilon_{Z}=0
$$

$$
\frac{\partial U}{\partial Z}+\frac{4}{Z}-a_{k} \frac{Z}{h^{2}}=0
$$

Интегрируя (6) с учетом граничного условия

$$
U=0 \text { при } r=R_{\text {вн.рез }}
$$

получим

$$
U=\frac{a_{k} \cdot Z}{h^{2}} \cdot\left(r-\frac{R_{\text {st.pes }}^{2}}{\kappa}\right)
$$

где $r$ - координат в радиальном направлении.

На первом этапе сжатия уплотнителя пакера происходит первое соприкосновение его наружной поверхности с внутренней поверхностью стенки эксплуатационной колонны. При этом необходимая осевая нагрузка может быть определена методом Ритца по выражению (3) с учетом, что $Q=Q_{1}, \Delta=\Delta_{1}$ т.е. $Q$-осевая необходимая для первого. Из условия первого соприкосновения и уравнения (8) с учетом: при $r=R_{k}, z=h$ тогда $u=\delta$ где $\delta=R_{k}-R_{\text {вн.рез. }}$ - зазор между наружной поверхностью (которой резина достигает внутренней поверхности колонны) уплотнительного элемента и внутренней стенкой эксплуатационной колонны, получим $R_{\text {н.рез }}=R_{\text {колон }}$

$$
\begin{aligned}
& Q_{1}=\frac{\frac{8 \pi}{3} G h \delta\left\{\left[\frac{R_{\kappa}^{2}-R_{\text {sн.p }}^{2}}{2}+\frac{R_{\text {sн. pes }}^{4}}{2}\left(\frac{1}{R_{\text {sн. } е \text { es }}^{2}}-\frac{1}{R_{k}^{2}}\right)\right]+\left(R_{k}^{2}-R_{\text {sн.рез }}^{2}\right)+\right.}{1} \times
\end{aligned}
$$

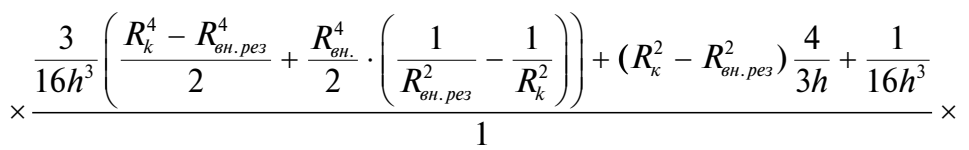

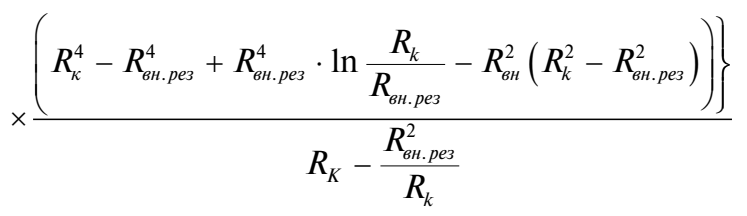


где соприкосновения наружной поверхности уплотнительного элемента с внутренней поверхности эксплуатационной колонны.

Минимум потенциальной энергии уплотнительного элемента достигается при условии Эйлера [2]:

$$
\frac{\partial \Pi}{\partial a_{k}}=0 ; \frac{\partial \Pi}{\partial \boldsymbol{B}_{\kappa}}=0
$$

Тогда из (3) с учетом (4), (8) и (9) получим выражение для определения постоянной $a_{\kappa}$.

$$
\begin{aligned}
& a_{k}=\frac{Q_{q} \cos ^{4} \alpha}{\frac{8 G \pi}{3 h}\left\{\left[\frac{R_{k}^{2}-R_{\theta H}^{2}}{2}+\frac{R_{\theta H}^{4}}{2}\left(\frac{1}{R_{\theta H}^{2}}-\frac{1}{R_{\kappa}^{2}}\right)\left(R_{\kappa}^{2}-R_{\theta H}^{2}\right)+\frac{3}{16 h^{3}}\right]\right.} \times \\
& \times \frac{\left.\left.\left(R_{\kappa}^{4}-R_{\theta H . p e s}^{4}\right)+R_{\theta H}^{4} \ln \frac{R_{k}}{R_{\theta H . p e s}}-R_{\theta H}^{2}\left(R_{\theta \kappa}^{2}-R_{\theta H}^{2}\right)\right]\right\}}{}
\end{aligned}
$$

$$
\begin{aligned}
& \text { Из условия первого соприкосновения и уравнения (4) с учетом }
\end{aligned}
$$

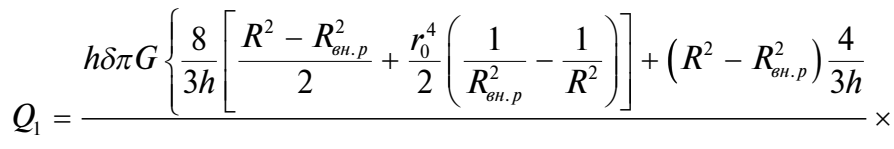

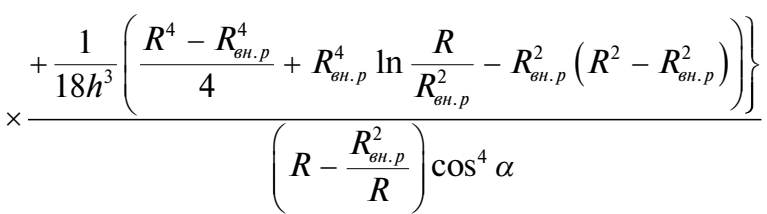

где $\delta$ - зазор между наружной поверхностью уплотнителя и внутренней стенкой корпуса сальника; $R$ - наружный радиус уплотнителя.

Из (12) видно, что первое соприкосновение наружной поверхности уплотнителя со стенкой эксплуатационной колонны происходит в его нижнем сечении, причем радиальная деформация наружной поверхности уплотнителя имеет линейный характер.

Дальнейшее увеличение осевой нагрузки приводит к деформации уплотнителя до соприкосновения его наружной поверхности по всей высоте со стенкой эксплуатационной колонны (рис. 1). При этом ради- 
альная деформация уплотнителя, удовлетворяющая граничному условию, может быть принята в виде

$$
U_{1}=b_{k} \frac{h-z}{h^{2}}\left(r-\frac{r_{0}^{2}}{r}\right)
$$

где $u_{1}$ - радиальная деформация уплотнителя; $b_{k}$ - постоянная, подлежащая определению.

Из условия несжимаемости (13) материала уплотнителя имеем

$$
W_{1}=b_{k} \frac{(h-z)^{2}}{h^{2}}
$$

где $W$ - осевая деформация уплотнителя.

Потенциальная энергия при дожатии уплотнителя до соприкосновения его наружной поверхности со стенкой эксплуатационной колонны по всей высоте с учетом осесимметричности деформации имеет вид:

$$
\Pi=2 \Pi G \int_{r_{0}}^{R} \int_{0}^{h}\left(\varepsilon_{r_{1}}^{2}+\varepsilon_{\theta_{1}}^{2}+\varepsilon_{z_{1}}^{2}+\frac{1}{2} \gamma_{r_{1}}^{2}\right) r \cdot d r d z-\Delta Q_{2}
$$

Из (15) с учетом (13), (14) и минимума потенциальной энергии имеем

$$
\begin{aligned}
& b_{k}=\frac{P_{2} \cos ^{4} \alpha}{2 \pi G\left\{\left[R_{\theta . p}^{2}-r_{\theta H . p}^{2}-r_{\theta H . p}^{4}\left(\frac{1}{r_{d y . h}^{2}}-\frac{1}{R^{2}}\right)\right]\right\}+3 h\left(R^{2}-R_{\theta H . p}^{2}\right)+} \times
\end{aligned}
$$

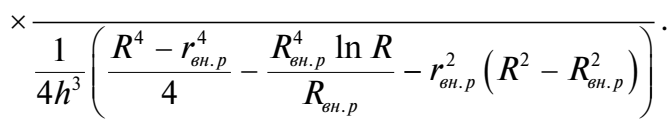

$P^{2}$ - осевая нагрузка, необходимая для сжатия уплотнителя до соприкосновения его наружной поверхности со стенкой эксплуатационной колонны по всей высоте.

Из условия $\left.U\right|_{\substack{z=h r \\ r=R}}=\delta$ получим с учетом (9) и (12) получим

$$
\begin{aligned}
& Q_{2}=\frac{\delta h \pi G\left\{\frac{8}{3 h}\left[\frac{R^{2}-r_{0}^{2}}{2}+\frac{r_{0}^{4}}{2}\left(\frac{1}{r_{0}^{2}}-\frac{1}{R^{2}}\right)\right]+\frac{4}{3 h}\left(R^{2}-r_{0}^{2}\right)+\right.}{\times} \\
& \times \frac{+\frac{1}{32 h^{3}}\left(\left(\frac{R^{4}-r_{0}^{4}}{4}+r_{0}^{4} \ln \frac{R}{r_{0}}-r_{0}^{2}\left(R^{2}-r_{0}^{2}\right)\right)\right\}}{\left(R-\frac{r_{0}^{2}}{R}\right) \cos ^{4} \alpha}
\end{aligned}
$$




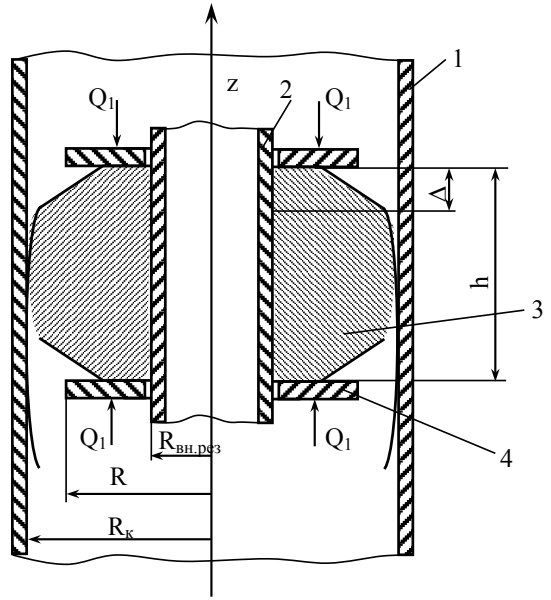

Рис. 1. Расчётная схема пакера

\section{1 - эксплуатационная колонна; 2 - ствол пакера;}

3 - уплотнительный элемент; 4 - шайба.

После полной деформации уплотнителя, когда его наружная поверхность полностью соприкасается со стенкой эксплуатационной колонны, необходимо еще добиться герметичности. Она достигается путем увеличения осевой нагрузки. При этом дифференциальное уравнение, описывающее равновесие элемента уплотнителя, с учетом несжимаемости его материала имеет вид [2]:

$$
\frac{d \sigma_{z}}{d z}+\frac{2 f}{R-r_{0}}=0
$$

где $\sigma_{z}$ - осевое напряжение в любом поперечном сечении уплотнителя; $f$-коэффициент трения между уплотнителем и стенками эксплуатационной колонны.

Из (18) имеем

$$
\sigma_{z}=\sigma_{z_{0}} \exp \left(-\frac{2 f z}{R-r_{0}}\right)
$$

где $\sigma_{z_{0}}$ - осевое напряжение в поперечном сечении уплотнителя в месте приложения силы $P_{3}$.

Для определения характера распределения удельного давления между наружной поверхностью уплотнителя и стенкой эксплуатаци- 
онной колонны необходимо суммировать эпюры его распределения на втором и третьем этапах.

На втором этапе, когда происходит сжатие наружной поверхности уплотнителя по всей его высоте до стенки корпуса, радиальное напряжение может быть определено так [2]:

$$
\sigma_{r_{1}}=2 G \varepsilon_{r_{1}}+S
$$

Из (20) с учетом $\left.\sigma_{r_{1}}\right|_{\substack{r=R \\ z=0}}=0$ и (13) имеем

$$
\begin{gathered}
\sigma_{r_{1}}=2 \frac{G \delta z}{\left(R-\frac{r_{0}^{2}}{R}\right) h}\left(1+\frac{r_{0}^{2}}{R^{2}}\right) \\
\sigma_{r}=\sigma_{r_{1}}+\sigma_{r_{2}}=\sigma_{z_{0}} \exp \left(-\frac{2 f z}{R-r_{0}}\right)+2 \frac{G \delta z}{\left(R-\frac{r_{0}^{2}}{R}\right) h}\left(1+\frac{r_{0}^{2}}{R^{2}}\right)
\end{gathered}
$$

Перейдем к рассмотрению характера распределения удельного давления между наружной поверхностью эксплуатационной колонны и внутренней стенкой уплотнителя, определяемого из (4), (9) и (15).

На первом этапе, когда происходит первое соприкосновение наружной поверхности уплотнителя со стенкой эксплуатационной колонны, удельное давление между поверхностью уплотнителя и со стенкой эксплуатационной колонны может быть определено как

$$
\sigma_{r_{0}}=\left.2 G \varepsilon_{r}\right|_{r=R_{\text {os.p }}}+S
$$

Из (23) с учетом (4) и условия $\left.\sigma_{r}\right|_{r=R}=0$ имеем

$$
\left.\sigma_{r}\right|_{r=R}=2 G \frac{a_{z}}{h^{2}}\left(1-\frac{R_{\theta н . p}}{R^{2}}\right)
$$

На втором этапе, когда происходит полное соприкосновение наружной поверхности уплотнителя с внутренней стенкой эксплуатационной колонны, удельное давление между поверхностями колона и уплотнителя определяется из (19) с учетом (9) и условия $\left.\sigma_{r}\right|_{\substack{z=o \\ r-r_{0}}}=0$

$$
\sigma_{r_{0}}^{*}=-4 B G \frac{z}{h^{2}}
$$

Суммарное удельное давление

$$
\left.\sigma_{r}\right|_{r=R_{\text {st.p }}}=2 G \frac{A_{z}}{h^{2}}\left(1-\frac{R_{\text {sH. }}}{R^{2}}\right)-4 B G \frac{z}{h^{2}}+\sigma_{z_{0}} \exp \left(-\frac{2 f z}{R-R_{\text {sH.p }}}\right)
$$


На рис. 2 представлены зависимости $\left[q_{1}\right]=Q_{1}$ и $\left[q_{1}\right]=Q_{2}\left[\mathrm{q}_{1}\right]=\mathrm{Q}_{2}$ от $\frac{R_{k}}{h}$ при $R$ вн.рез. Как видно из рисунка 2 , с ростом $\frac{R_{k}}{h}$ значения $\left[q_{1}\right]$ и $\left[q_{2}\right]$ уменьшаются.

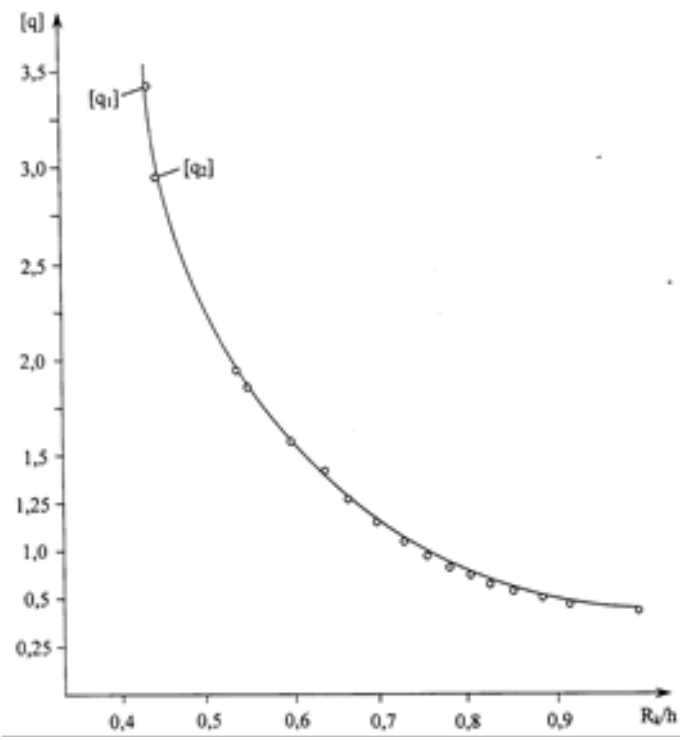

Рис. 2. Зависимость безразмерного удельного давления уплотнителя пакера от безразмерных диаметрических параметров

Таким образом, полученные зависимости (16) и (17) показывают, что равномерного распределения удельного давления между наружной поверхностью уплотнителя и стенкой эксплуатационной колонны, наряду с другими факторами многое зависит от уплотняемого зазора $\sigma$.

\section{3. Исследование осложнения нагруженности уплотнителя} пакеров с применением штангового глубинного насоса

Основную роль в герметизации пакерных устройств (с применением штангового глубинного насоса) выполняет уплотнительный элемент, который принимает на себя избыточное давление, действия 
среды и температуры, различных видов нагружения его при посадке и в процессе уплотнения (работу). Из практики известно, что действия осевых сил и давления часто нарушают герметичность упражнения. Особенно при работе пакера с ШГН (штангового, глубинного насоса) возникает дополнительное динамическое усилие, которое предается уплотнителем пакера [3; 4]. В некоторых случаях эксплуатации это равновесие нарушается, так как некоторые из сил нейтрализуются условиями работы труб или их закреплением пакерами.

Рассмотрим наиболее часто встречающиеся случаи возникновения осевых сил. При этом отсутствующие силы, приводящие к нарушению равновесия, будем брать с обратным знаком [7].

I. Концы труб закреплены с пакером и на устье. Днище снизу отсутствует и $\mathrm{P} \neq 0, P_{H}=0$. В этом случае растягивающее кольцевое напряжение $\sigma_{k}=P_{B} \cdot d / 2 \sigma$ вызывает в защемленных трубах растягивающее осевое усилие, которое передается в уплотнители пакера:

$$
N_{1}=\sigma_{k} \mu S
$$

Подставляя в (27) значения $\sigma_{k}=P_{B} \cdot d / 2 \sigma, S=\pi d_{c p} \sigma ; F=\pi d^{2} / 4$ (площадь в свету) и принимая $d_{c p}=d$, получим

$$
N_{1} \approx 2 P_{B} \cdot F_{B} \cdot \mu
$$

где $\mu$-коэффициент Пуассона так как нижний конец не заглушен, то следует взять со знаком минус. Суммарные знаки сжимающие усилия, действующие внизу, в месте заделки пакера:

$$
N_{R 1}=2 P_{B} \cdot F_{B} \cdot \mu-P_{B} F_{B}
$$

Усилие $N_{R 1}$ будет сжимающим и момент вызывает продольный изгиб.

II. То же закрепление но $P_{B}=0, P_{H}=0$

В этом случае

$$
\sigma_{k}=-P_{H} \cdot D / 2 \sigma \text { и } N_{2}=-2 P_{H} \cdot \mu F_{H}
$$

где $F_{H}=\frac{\pi D^{2}}{4}=0,785 D^{2}$

$F_{H}-$ площадь сечения трубы по наружному ее диаметру.

Поскольку недостающая до равновесия сжимающая сила $\left(-P_{H} \cdot F_{H}\right)$ на днище отсутствует, берем ее с обратным знаком. Результирующая осевая сила после аналогичных преобразований

$$
N_{R 2}=P_{H} \cdot F_{H}(1-2 \mu)
$$


III. То же закрепление, но $P_{b} \neq 0 ; P_{H} \neq 0$ Р. В аналогично рассмотренном случае получим

$$
N_{R 3}=(1-2 \mu) \cdot\left(P_{H} \cdot F_{H}-P_{B} \cdot F_{B}\right)
$$

IV. Tо же закрепление $P_{H}=P_{B}=0$, но внутри труб находится жидкость с плотностью. Действие жидкости на трубы может быть приравнено действию среднего давления

$$
\left(-P_{H} \cdot F_{H}\right) N_{R 2}=P_{H} \cdot F_{H}(1-2 \mu)
$$

$P_{H}=P_{B}=0$, но внутри труб находится жидкость с плотностью $\rho_{B}$. Эпюра давления жидкости на трубы. Действие жидкости на трубы может быть приравнено действию среднего давления

$$
P_{c p}=\rho_{B} g H / 2
$$

Тогда аналогично рассмотренному выше при значении $\mu=0,28$ получим

$$
N_{R 4}=-0,72 \rho_{B} g H F_{B}
$$

V. При действии давления жидкости. как изнутри, так и снаружи НКТ (при $\mu=0,28$ ):

$$
N_{R 5} \approx 0,44\left(P_{H} \cdot F_{H}-P_{B} \cdot F_{B}\right)+0,72 g H \cdot\left(\rho_{H} F_{H} \rho_{B} F_{B}\right)
$$

VI. Вес столб жидкости при движении плунжера вверх снимается с труб и передается на плунжер. При этом равновесие нарушается, так как растягивающая сила, снимаемая с труб и создающая опасность продольного изгиба,

$$
N_{R 6}=-\rho_{\varkappa} \cdot g \cdot H \cdot F_{H}
$$

где $-F_{H}$ площадь плунжера.

При диаметре плунжера $D_{I}, F_{I}=\pi D_{\Pi}^{2} / 4$

\section{4. Факторы, определяющие герметизирующую способность уплотнения}

Степень деформации уплотнительного элемента должна быть достаточной, чтобы удержать уплотнительные элементы от вытяжки из места установки-посадки при действии на него давления герметизируемой среды и для обеспечения герметичности при действии того же давления осевой деформации.

В месте (относительно) посадки под шайбой уплотнительного элемента пакера будет 


$$
\varepsilon_{O_{A}}=\frac{\left(h_{0}-h_{1}\right)}{h_{O}}
$$

В месте сжатия боковой части уплотнительного элемента по высоте $\mathrm{B}_{1}$ деформации сжатия:

$$
\varepsilon_{\text {бок }}=\left(h-h_{1}\right) / h_{1}
$$

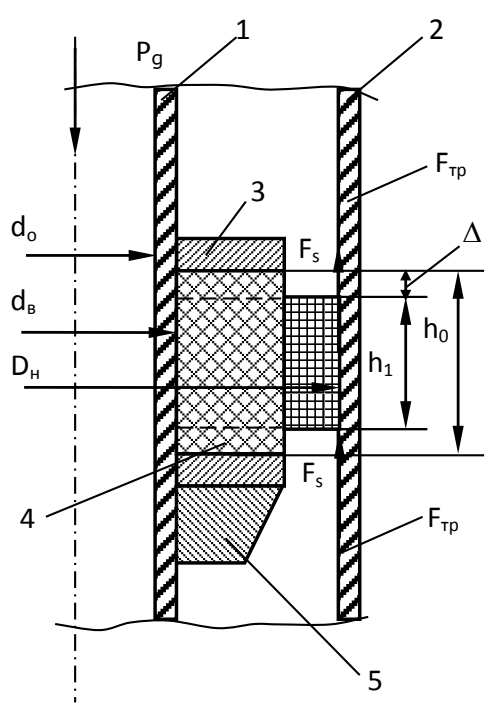

Рис. 3. Расчётная схема уплотнительного элемента пакера:

1 - ствол; 2 - эксплуатационная колонна; 3 - опорная шайба;

4 - уплотнительный элемент; 5 - конус

Начальное контактное напряжение, созданное при деформации уплотнительного элемента, может быть найдено из уравнения [6]

$$
\sigma_{0}=E\left(1+2 \mu_{m p} \Phi\right) \frac{\varepsilon_{o c}}{\varepsilon_{\text {бок }}}
$$

где $\Phi$ - фактор формы, равный:

$$
\Phi=\frac{S_{\text {оп }}}{S_{б о к}}=\frac{D_{c н . \kappa}^{2}-D^{2}}{4 D_{\text {вн }} \cdot h_{0}}
$$

Условием обеспечения устойчивости уплотнения, действующего на тело уплотнительного элемента избыточного давления Р, является 


$$
E_{m p}=P_{\Delta P} S_{e \phi \phi}
$$

где $S_{\text {eфф }}$ - эффективная площадь уплотнения, т.е. величина характеризующая способность уплотнительного элемента преобразовывать давление в осевую силу:

$$
S_{e \phi \phi}=\frac{\Delta Q}{\Delta P}
$$

где $\Delta Q$ - изменение усилия в уплотняемой поверхности в контакте с уплотнением.

$\Delta P$ - изменение давления в скважине (над или под пакера).

$\Delta P_{\Delta P}$ - давление, необходимое для осуществления применения уплотнителя контактных поверхностей.

Тогда полное перемещение уплотнительного элемента при проявление самоуплотнения будет:

$$
\Delta=\Delta_{0}+\Delta_{P}
$$

Также сила трения на поверхностях контакта уплотнительного элемента составляет:

$$
\begin{aligned}
F_{m p}= & \mu_{m p} \cdot F=\mu_{m p} \cdot \sigma \cdot \pi \frac{D_{\theta . \kappa .}^{2}-D^{2}}{4}=\frac{\pi}{4} \cdot \mu_{m p} \cdot E . \\
& \left(1+\mu_{m p} \cdot \frac{D_{\theta . \kappa}^{2}-D^{2}}{4}\right) \cdot\left(D_{\theta H . \kappa}^{2}-D^{2}\right)\left(\frac{h_{2}-h_{1}}{h_{2}}\right)
\end{aligned}
$$

Из условия устойчивости с учетом значения эффективной площади уплотнения найдем значение давления $P_{\Delta P}$, вызывающего полное перемещение уплотнения, при котором уплотнитель не вытягивается из места установки (посадки):

$$
P_{\Delta P}=\frac{\mu_{m p} E\left(1+\mu_{m p} \cdot \frac{\mathrm{D}_{\text {внк }}^{2}-\mathrm{D}^{2}}{\mathrm{D}_{\text {внк }} \cdot \mathrm{h}_{\mathrm{o}}}\right)\left(D_{\text {внк }}^{2}-\mathrm{D}^{2}\right)\left(h_{0}-h_{2}\right)}{h_{2} \cdot\left(D^{2}+D d+d^{2}\right)}
$$

При действии указанного давления $P_{\Delta P}$ уплотнителем элемента герметизация обеспечивается благодаря проявлению эффекта самоуплотнения в результате увеличения контактного напряжения до значения $\sigma^{*}$,

$$
\sigma^{*}=\sigma_{0}+\alpha P_{\Delta P}
$$

где $\alpha$ - коэффициент передачи давления, для уплотнения пакера $\alpha=0,85 \ldots 0,95$. 
Условие герметичности соединения выражается зависимостью:

$$
\sigma^{*} \geq P_{\Delta P}
$$

Сохранение герметичности соединения при температуре и в процессе длительной эксплуатации связано с уменьшением начального напряжения вследствие изменения свойств резины (уплотнению) в этих условиях [6]:

$$
\sigma(t, \tau)=\sigma_{o} K \beta
$$

где $K_{s}$ - коэффициент восстанавливаемости резины при температуре:

$$
K_{6}=\frac{h_{1}-h}{h_{0}-h}
$$

$h_{1}$ - размер восстанавливающегося уплотнителя после снятия нагрузки при данной температуре:

$\beta$ - относительная остаточная эластичность резины [6]

где $H=\frac{E_{o c m}}{E}=\frac{\mathrm{h}_{0}-h_{2}}{h_{0}-h_{1}}$

$$
\beta=1-H
$$

$h_{2}$ - высота уплотнителя после снятия нагрузки

Еост - остаточная деформация : $E_{\text {ост }}=\left(\frac{\mathrm{h}_{0}-h_{2}}{h_{0}-h_{1}}\right)$

Тогда из (12) получим:

$$
\sigma(t, \tau)=\sigma_{0} K_{b} \cdot \beta+\alpha \cdot P_{\Delta P}
$$

Или, подставляя значение $\sigma_{0}$ и $P_{\triangle P}$, после преобразования, получим:

$$
\sigma^{*}(t, \tau)=E\left(1+\mu_{m p} \frac{\mathrm{D}_{\text {вн.к }}^{2}-\mathrm{D}^{2}}{2 D_{\text {вн.к }} \mathrm{h}_{0}}\right) \cdot\left(\frac{h_{0}-h_{2}}{h_{2}}\right) K_{\theta} \cdot \beta+\alpha \cdot \mu_{m p} \cdot \frac{\mathrm{D}_{\text {вн.К }}^{2}-\mathrm{D}^{2}}{\mathrm{D}^{2}+D \cdot d+d^{2}}
$$

\section{5. Выводы}

1. Разработана методика расчета параметров герметизации уплотнительных узлов пакера.

2. Установлено что, равномерное распределение удельного давления уплотнителя пакера зависит от уплотняемого зазора.

3. Полученное аналитическое выражение позволяет по заданной характеристике резины рассчитать продолжительность сохранения герметичности при давлений $P_{\Delta P}$, уплотнения пакера (модулю Е, коэффициенту $K_{6}$ восстанавливаемости при температурах, относительной остаточной эластичности коэффициенту трения $\mu_{K}$ ) и геометрическим параметрам уплотнению пакера и его пакерующего узла. 


\section{Список литературы:}

1. Бидерман В.Л., Сухова Н.А. Монтаж, эксплуатация и ремонт скважинных штанговых насосных установок : Справочник. Баку : Элм, 1993. 208 с.

2. Мамедов В.Т., Ханкишиева Т.У. Определение оптимальной формы уплотнения сальника штока штанговых скважинных насосов. Оборудование и технологии для нефтегазового комплекса. Москва, 2015. С. 31-33.

3. Песляк Ю.А. Расчет напряжений в колоннах труб нефтяных скважин. Москва : Недра, 1973. 216 с.

4. РДО653-39-72. Методика расчета проектирования уплотнительных узлов пакеров, обеспечивающих эффект самоуплотнения. Баку: ОКБ Нефтемаш, 1992. 18 с. (Мамедов В.Т., Гурбанов Р.С.).

5. Протураев В.Н., Дырда В.И. Резиновые детали машин. Изд. 2-ое перераб. и доп. Москва : «Машиностроение», 1977. 216 с.

6. Зайцев Ю.В., Максутов Р.А., Асфандияров Х.А. Оборудование для предотвращения открытых фонтанов нефтяных и газовых скважин. Москва : Недра, 1973. 224 с.

7. Касумова С.А., Гусейнли 3.С., Мамедов Г.В. Исследование осложнения нагруженности уплотнителя пакеров при посадке и в процессе уплотнения его с применением штангового глубинного насоса. Известия высших технических учебных заведений Азербайджана. Баку, 2012. № 2(78). C. 19-22.

8. Мамедов В.Т., Асланов Дж.Н., Байрамов Ф.Г., Гусейнли З.С. Методика расчета параметров герметизации уплотнительного узла пакера применяемых при ремонте скважин. Global Sciense and Innovation Materials of the VIII International Scientific Conference, JUNE 22 ${ }^{\mathrm{ND}}-23^{\mathrm{RD}}, 2016$.

9. Гусейнли 3.С. Факторы, определяющие герметизирующую способность уплотнения пакерного оборудования. Оборудование и технологии для нефтегазового комплекса. Москва : ВНИИОЭНГ, 2015. № 2. С. 25-28.

\section{References:}

1. Biderman V.L., Sukhova N.A. Installation, exploitation and repair of downhole sucker pumping units: Directory. Baku: Elm, 1993. 208 p.

2. Mamedov V.T., Khankishieva T.U. Determination of the optimal sealing shape of the stem seal of sucker rod pumps. Equipment and technologies for the oil and gas complex. Moscow, 2015. Pp. 31-33.

3. Peslyak U.A. Calculation of stresses in pipe columns of oil wells. Moscow: Nedra, 1973. 216 p.

4. RDO 653-39-72. The calculating methodology for the design of sealing packer knots providing the effect of self-sealing. Baku: DS Neftemash, 1992. 18 p. (Mamedov V.T., Gurbanov R.S.).

5. Proturaev V.N., Dyrda V.I. Rubber parts of machines. Ed. 2nd rebuild. and add. Moscow: "Engineering", 1977. 216 p.

6. Zaitsev U.V., Maksutov R.A., Asfandiyarov H.A. Equipment for the prevention of open fountains of oil and gas wells. Moscow: Nedra, 1973. 224 p. 
7. Kasumova S.A., Guseynli Z.S., Mammadov G.V. Research of complication og packers compactor loading at itz landing and sealing process by application of deep rod pumps.News of Azerbaijan high technical educational institutions. Baku, Azerbaijan, 2012. № 2(78), pp. 19-22.

8. Mammadov V.T., Aslanov J.N., Bayramov F.Q., Huseynli Z.S. Calculation method of hermetic seal assembly parameters of the packer used during repairing wells. Global Sciense and Innovation Materials of the VIII International Scientific Conference, JUNE 22 $2^{\mathrm{ND}}-23^{\mathrm{RD}}, 2016$.

9. Huseynli Z.S. Faktors that detemine packing abilitu of packer equipment seal. Equipment and technologies for oil and qaz complex. Moskow: VNIIOENG, 2015. № 2 . 